\title{
FUNCIONALIDAD FAMILIAR, CONDUCTAS INTERNALIZADAS Y RENDIMIENTO ACADÉMICO EN UN GRUPO DE ADOLESCENTES DE LA CIUDAD DE BOGOTÁ
}

\author{
FAMILY FUNCTIONALITY, INTERNALIZED BEHAVIOR AND ACADEMIC \\ ACHIEVEMENT IN A GROUP OF ADOLESCENTS BOGOTA CITY \\ JAIME Humberto MORENO MÉNDEZ*, KAREN LIZETH ECHAVARRÍa LlANO, ANDREA PARDO, \\ YOHANA QUIÑNONES \\ UNIVERSIDAD DE SAN BUENAVENTURA, BOGOTÁ - COLOMBIA
}

FECHA RECEPCIÓN: 07/04/2014 • FECHA ACEPTACIÓN: 04/08/2014

Para citar este artículo: Moreno, J., Echavarría, K., Pardo, A., \& Quiñones, Y. (2014). Funcionalidad familiar, conductas internalizadas y rendimiento académico en un grupo de adolescentes de la ciudad de Bogotá. Psychologia: avances de la disciplina, $8(2), 37-46$

\begin{abstract}
Resumen
El presente estudio tuvo como objetivo describir la asociación entre la funcionalidad familiar, las conductas internalizadas y el rendimiento académico en un grupo de adolescentes de un colegio público de la ciudad de Bogotá, Colombia. El estudio fue de tipo descriptivo con un método de asociación, empleando como instrumentos las listas de chequeo de la conducta infantil de Achenbach y Edelbrock (1987), el de autorreporte (YSR), el cuestionario de padres (CBCL) y el cuestionario de profesores (TRF). También se empleó el APGAR familiar de Smilkstein (1978) y se revisó el boletín académico de los estudiantes. Los participantes fueron 63 adolescentes entre 12 y 16 años de edad, sus respectivos padres y profesores. Dentro de los resultados encontrados se estableció que la mayoría de los adolescentes reportan una disfunción familiar moderada y un rendimiento académico medio; adicionalmente, se evidenció que aproximadamente un $19.56 \%$ de la población presenta conductas internalizadas, siendo el puntaje más alto el reportado por los padres de familia.

Palabras clave: funcionalidad familiar; conductas internalizadas; rendimiento académico; adolescente.
\end{abstract}

\begin{abstract}
The present study aimed to describe the association between family functioning, internalized behavior and academic achievement in a group of adolescents from a public school in the city of Bogota Colombia. The study was descriptive with a method of association, using as instruments checklists of child behavior Achenbach and Edelbrock (1987), the self-report (YSR), the parent questionnaire (CBCL) and the teacher questionnaire (TRF). It also was used the family APGAR Smilkstein (1978) and revised the student academic newsletter. Participants were 63 adolescents between 12 and 16 years old, their parents and teachers. Among the results established that the majority of adolescents reported family dysfunction moderate and average academic achievement, additionally, showed that approximately $19.56 \%$ of the population has internalized behaviors, being the highest score reported by the parents family.

Keywords: Family functionality; internalized behavior; academic achievement; adolescents.
\end{abstract}

* Correo electrónico: jaimehm@yahoo.com. Grupo de Investigación Avances en Psicología Clínica y de la Salud. Universidad de San Buenaventura, Bogotá. 


\section{Introducción}

El desarrollo de los adolescentes se construye a partir de las relaciones significativas que se suceden al interior de la familia y que posteriormente se fortalecen en el entorno escolar. Cuando se presentan dificultades en alguno de estos ambientes, se pueden configurar problemas de ajuste de tipo externalizado e internalizado en el adolescente e impactar su rendimiento académico y por ende interferir en las interacciones constructivas entre padres e hijos.

A lo largo del tiempo, diversos estudios comprueban que la familia configura un aspecto esencial en la formación de los individuos (Betancourt, \& Andrade, 2011; Moreno, \& Chauta, 2012; Viola, 2010), ya que es un escenario en el que se promueve la socialización, el afecto, el desarrollo y la realización personal en diversas etapas de la vida de sus miembros. En el proceso hacia la adultez, la familia forma parte de la construcción de la autoestima y el sentido de sí mismo desde los valores que allí se aprenden (Palacios, \& Rodrigo, 1998).

Ahora bien, Forero, Avendaño, Duran, Duarte y Campo (2006), proponen que la familia es adicionalmente una unidad biopsicosocial que propende por la salud de todos sus miembros, por medio de la transmisión de creencias y valores de padres a hijos, cuya preocupación es la salud y el bienestar integral de sus integrantes; por lo que es innegable la influencia de esta sobre los factores de riesgo y protección en los adolescentes (Pérez, PinzónPérez y Alonso, 2007).

En relación con lo anterior, Smilkstein (1978), asegura que una familia funcional es aquella que logra promover el desarrollo integral de sus miembros, así como un estado de salud favorable en ellos, donde cada uno de sus integrantes manifieste una satisfacción con el cumplimiento de los parámetros básicos de la función familiar, como son, adaptación, participación, ganancia o crecimiento, afecto y recursos.

Cuando los miembros enfrentan problemáticas complejas que obstaculizan su desarrollo y la salud de sus integrantes, es cuando se ve comprometida su funcionalidad familiar. La adolescencia es una de las etapas de la vida en donde más se producen inconvenientes dado que, debido a los cambios propios de la edad a nivel emocional y comportamental, se genera una inestabilidad que produce malestar (Moreno, \& Chauta, 2012); lo que da como resultado que algunos padres o tutores asuman posiciones cuya base es el uso de la violencia para afrontar el conflicto y ejercer el poder mediante la fuerza física, lo que afecta el comportamiento y el desempeño escolar de sus hijos (Hewitt, Moreno, Oliveros, \& Pardo, 2010).

Los adolescentes denotan una mayor satisfacción familiar cuando forman parte de familias con buena comunicación, un grado alto de adaptabilidad y una fuerte vinculación emocional entre sus miembros (Jiménez, Musitu, \& Murgui, 2005).

Gladding (1995) señala que una familia saludablefuncional ideal tiene características como ser una fuente legítima de autoridad, estabilidad y apoyo a lo largo del tiempo, mostrar una suficiente flexibilidad y adaptabilidad para acomodarse a los cambios normales del desarrollo, así como a las crisis inesperadas. También, están conformadas por individuos motivados y comprometidos con la familia, tienen adecuados patrones de comunicación, donde estimulan la participación activa de los niños sobre las decisiones que les afectan. Igualmente están socialmente conectadas con otras familias, tienen un alto grado de orientación religiosa y espiritual, son fuente de información y transmisión de valores y son capaces de enfrentar las crisis de una manera positiva, de hecho proveen refugio y lugar de consuelo para recuperarse de los esfuerzos, tensiones y problemas que hayan supuesto costes para algunos de sus integrantes.

Otro escenario de socialización de vital importancia para los adolescentes, es el colegio, el cual contribuye a la comprensión, el aprendizaje y la expresión de creencias y valores de la sociedad, que impactan en su desempeño académico.

Jiménez (2000), define el rendimiento académico como el nivel de conocimientos que muestra un estudiante en un área o materia, el cual se determina con base en la edad y el nivel académico. Portellano (1989), distingue dos tipos de rendimiento académico, el rendimiento suficiente y el satisfactorio. El primero corresponde a la medición de las aptitudes pedagógicas, conocimientos escolares, por medio de las calificaciones; el segundo por su parte, corresponde a la capacidad intelectual del niño tomando como punto de referencia la relación existente entre sus capacidades intelectuales y el aprovechamiento que el niño evidencia de las mismas (Hewitt et al., 2010; Moreno, \& Chauta, 2012). 
Existen diferentes explicaciones por las cuales el adolescente puede presentar un bajo rendimiento académico. Dependiendo del momento de su aparición, se puede clasificar en primario o secundario. El primario, hace referencia a dificultades madurativas del sistema nervioso por lo que se presenta en los inicios de la escolaridad y dado que es una alteración estructural, puede persistir si no se corrige. El otro tipo de bajo rendimiento académico, el secundario, está asociado a dificultades emocionales y suele aparecer en los últimos grados de escolaridad, en donde los estudiantes adolescentes presentan cambios propios de dicha etapa fracasando en sus estudios (Portellano, 1989).

Los problemas emocionales y afectivos en la adolescencia conllevan la necesidad de comprender sus causas y dar respuestas a dichas dificultades. En Colombia, según el último Estudio Nacional de Salud Mental del Adolescente (2010), el 16,1 \% de los adolescentes ha sufrido o sufre uno o más trastornos mentales. Siendo mayor esta proporción para las adolescentes con un $17,4 \%$ y un 14,8 \% para los adolescentes del sexo masculino. En su orden, la mayor prevalencia se encontró en ansiedad con un $5.5 \%$, depresión mayor con $5 \%$ y los problemas de comportamiento negativista desafiante con un $2 \%$ (Ministerio de Protección Social, Universidad CES y la Oficina de Naciones Unidas contra la Droga y el Delito - UNODC, 2010).

En las últimas décadas, varios clínicos e investigadores interesados en el estudio de los trastornos psicológicos de la infancia y la adolescencia han considerado el modelo teórico conocido como psicopatología evolutiva o del desarrollo (Buckholdt, Parra, \& Jobe-Shields, 2014; Hewitt, Moreno, Oliveros, \& Pardo, 2010; McCullough, \& Shaffer, 2014; Moreno, \& Chauta, 2012). En este enfoque, tanto la conducta normal como la anormal se entienden como variaciones en un continuo de rasgos o características y no como fenómenos dicotómicos (Lemos, 2003).

Achenbach y Rescorla (2001), consideran que las conductas desviadas son variaciones cuantitativas de las características que pueden ser normales en ciertos periodos del desarrollo, por lo que identificaron ocho escalas denominadas de banda estrecha o síndromes de primer orden y tres dimensiones de segundo orden o banda ancha. Los síndromes de primer orden son depresión/retraimiento, quejas somáticas, depresión/ansiedad, problemas sociales, problemas de pensamiento, problemas de atención, conducta agresiva y conducta delictiva. Las dimensiones de segundo orden hacen referencia a una dimensión Internalizante, una Externalizante y otra Mixta.

La dimensión Internalizante implica la vivencia de tensión psicológica en el propio sujeto, síntomas de angustia, depresióny estados alterados deánimo, yagruparía los síndromes depresión/ retraimiento, quejas somáticas y depresión/ansiedad. La dimensión Externalizante está relacionada con síntomas de agresividad, déficit de atención, hiperactividad, conducta desorganizada, y agruparía los síndromes de conducta delictiva y conducta agresiva (Achenbach, 1985; Achenbach, \& Rescorla, 2001).

Los comportamientos internalizados son un subgrupo de la psicopatología infantil que involucra disturbios emocionales. Las alteraciones de las emociones o síndromes internalizados están relacionadas con inestabilidad del estado de ánimo, obsesiones, problemas somáticos, nerviosismo, inseguridad, miedos, fobias, tristeza, apatía, disforia, inquietud, tensión, preocupación y culpabilidad, entre otros síntomas. Estas dificultades son el resultado de una interacción compleja entre factores ambientales y características de los adolescentes, por tal motivo la presente investigación tiene como objetivo describir la asociación entre el nivel de funcionalidad familiar, las conductas internalizadas y el rendimiento académico en un grupo de adolescentes de un colegio distrital de la localidad de Usaquén en la ciudad de Bogotá.

\section{Método}

\section{Tipo de estudio}

Este estudio fue de corte empírico-analítico de tipo descriptivo con un método de asociación, ya que se buscaba describir la relación que existía entre la funcionalidad familiar, las conductas internalizadas y el rendimiento académico.

\section{Participantes}

Dentro de la investigación participó un grupo de 63 adolescentes, 34 de ellos de sexo femenino y 29 de sexo masculino, con edades entre los 12 y 16 años, de los grados octavo y noveno en la jornada mañana y tarde, seleccionados al azar. También participaron 63 padres de familia correspondientes a cada uno de los adolescentes de la muestra y 4 profesores de un colegio distrital, ubicado en la localidad de Usaquén en la ciudad de Bogotá Colombia. 


\section{Instrumentos}

Lista de chequeo de la conducta infantil de Achenbach, \& Edelbrock (2001), formato de padres (CBCL): fue diseñada en respuesta a la necesidad de procedimientos estandarizados para registrar los comportamientos relevantes de los jóvenes. El índice de confiabilidad es de 0.985. La validez del contenido del CBCL se refiere a si sus ítems se relacionan con las preocupaciones clínicas de padres y trabajadores en salud mental, representados en las distintas escalas de la prueba. Se encontró que 116 de los 118 ítems de problemas de conducta y todos los 20 ítems de competencia social se asociaron significativamente con el estado clínico $(\mathrm{p}<.01)$. Esta lista ha sido utilizada en estudios transculturales y aplicada en niños colombianos (Henao, \& Mahecha, 2005; Hewitt, Jaimes, Vera, \& Villa, 2012; Rescorla et al., 2012).

Lista de chequeo de la conducta infantil de Achenbach, \& Rescorla (2001), formato de autorreporte (YSR): fue diseñada para obtener información acerca de las competencias y problemas de jóvenes. Confiabilidad. La correlación de Pearson para el grupo de 11 a 14 años fue de 0.77 y para el grupo de 15 a 18 años, fue de 0.89 .

Lista de chequeo de la conducta infantil de Achenbach, \& Edelbrock (2001), formato de profesores (TRF): Fue diseñada a partir de la CBCL para ser respondida por profesores que conozcan a los niños y jóvenes por un tiempo mínimo de dos meses. El índice de confiabilidad es de 0.46 para la de internalización.

Los tres instrumentos CBCL, YSR y TRF se califican con base en la escala de $0=$ No es cierto, $1=$ Un poco cierto o a veces, 2 = Es verdad o frecuentemente es verdad. Para la calificación de la escala se suman los puntajes en cada una de las subescalas y luego se transforman en puntuaciones T. Cuando este puntaje normalizado es igual o superior a 70 se puede decir que el sujeto presenta un indicador clínico.

APGAR Familiar: Fue diseñado por Smilkstein (1978) para aproximarse de una manera objetiva a la función familiar del paciente. Consiste en una tabla con cinco enunciados que hacen referencia a: Adaptación, Participación, Crecimiento, Afecto y Resolución. Cada uno de los enunciados se evalúa en una escala de cinco opciones: Siempre $=4$, Casi siempre $=3$; Algunas veces $=2$; Casi nunca $=1 ;$ Nunca $=0$. El formato lo debe diligenciar directamente el paciente y la interpretación del resultado es: Buena función familiar: 18-20 puntos; Disfunción familiar moderada: 10-17 puntos; y Disfunción familiar severa: 9 puntos o menos

Boletín académico: las notas que se manejan en el colegio fueron agrupadas de la siguiente manera, de 2.0 a 2.9 se estableció un nivel bajo de rendimiento académico, de 3.0 a 3.9 se estableció un nivel medio de rendimiento académico y de 4.0 a 5.0 se estableció un nivel alto de rendimiento académico.

\section{Procedimiento}

Fase de inicio: se realizó una solicitud por escrito a la institución educativa con el fin de pedir su aprobación para realizar la investigación; además de esto se realizó el muestreo de la población para obtener el $\mathrm{N}$ con el cual se trabajó.

Para el desarrollo de este proyecto, se tuvo en cuenta la participación voluntaria de padres, maestros y adolescentes. Esto se hizo a partir de un documento donde dichos participantes dieron su consentimiento para participar en la investigación. Para esto se utilizaron los formatos de consentimiento y asentimiento informado. Finalmente, al dar por terminada la aplicación de las pruebas, se desarrolló un taller llamado «autoestima y comunicación» para los estudiantes como agradecimiento a la participación dentro de la investigación. Para la aplicación de este taller se contó con la participación de los integrantes del semillero de investigación de la línea en la cual se desarrolla este estudio, estos estudiantes fueron previamente entrenados para realizar la aplicación del taller.

Fase de implementación: se realizó la aplicación del formato de autorreporte, formato para padres y formato para profesores de la lista de chequeo de la conducta infantil y del APGAR familiar, explicando en términos simples el objetivo de la investigación, finalmente, se revisaron los boletines escolares con el fin de obtener información acerca de las calificaciones de los estudiantes, luego de aplicar los instrumentos se procedió a organizar y analizar la información.

Fase de finalización: a partir de los resultados obtenidos mediante el programa SPSS, se realizó una discusión. 


\section{Resultados}

Los resultados se presentan dando respuesta a los objetivos del estudio, en ese sentido primero se hace referencia al análisis descriptivo de la funcionalidad familiar, el rendimiento académico y el comportamiento internalizado, este último desde la perspectiva de los adolescentes (YSR), los padres (CBCL) y los profesores (TRF).

Posteriormente se presentan las principales asociaciones encontradas entre las variables funcionalidad familiar, rendimiento académico y comportamiento internalizado. Para esta investigación se tomó como puntaje significativo del chi- cuadrado de Pearson aquellos puntajes inferiores a .05 .

\section{Análisis descriptivo}

De acuerdo con lo hallado en el APGAR Familiar, el $39.7 \%$ de los adolescentes tienen un núcleo familiar funcional, el $44.4 \%$ presenta disfuncionalidad moderada y finalmente el $15.9 \%$ presenta disfuncionalidad severa. En cuanto al rendimiento académico, el $15.9 \%$ de los adolescentes se encuentra en un nivel alto, el $71.4 \%$ tiene un nivel medio y el $12.7 \%$ se encuentra en un nivel bajo.

En relación con las conductas internalizadas, de acuerdo con el autorreporte YSR de los propios adolescentes, se encontró que el $14 \%$ evidencia problemas conductuales de este tipo. Según el reporte dado por los padres de familia acerca de las conductas internalizadas de sus hijos adolescentes mediante la CBCL, se encontró que en un $31.7 \%$ de la muestra se evidencian problemas conductuales de este tipo. Por otro lado, los profesores reportan a partir del formato TRF, que un $13 \%$ los presenta.

\section{Análisis comparativo}

Al realizar el cruce de las variables se encontraron las siguientes asociaciones entre la funcionalidad familiar y la conducta internalizada medida según el YSR, el CBCL y el TRF descritas a continuación:

Como se aprecia en la tabla 1 , con respecto a las asociaciones entre el rendimiento académico y el autorreporte de los adolescentes de la conducta internalizada, un $9.5 \%$ de los que las presenta tienen un nivel académico medio mientras que un $3.2 \%$ tienen un nivel académico bajo.
Tabla 1. Asociación entre el rendimiento académico y las conductas internalizadas (YSR)

\begin{tabular}{cccc}
\hline \hline & \multicolumn{2}{c}{ Conductas internalizadas } \\
\cline { 3 - 4 } & & $\begin{array}{c}\text { Sin } \\
\text { indicador } \\
\text { clínico }\end{array}$ & $\begin{array}{c}\text { Con } \\
\text { indicador } \\
\text { clínico }\end{array}$ \\
\hline \hline \multirow{2}{*}{$\begin{array}{c}\text { Rendimiento } \\
\text { académico }\end{array}$} & Alto & $14.3 \%$ & $1.16 \%$ \\
& Medio & $63.5 \%$ & $9.5 \%$ \\
\hline \hline
\end{tabular}

Por otro lado, en la tabla 2 se encuentra que un $3.2 \%$ de la población adolescente con conductas internalizadas presenta una disfunción familiar moderada y otro $3.2 \%$ evidencia disfunción familiar severa.

Tabla 2. Asociación entre la funcionalidad familiar y las conductas internalizadas (YSR)

\begin{tabular}{cccc}
\hline \hline & & \multicolumn{2}{c}{$\begin{array}{c}\text { Conductas } \\
\text { internalizadas }\end{array}$} \\
\cline { 3 - 4 } & & $\begin{array}{c}\text { Sin } \\
\text { indicador } \\
\text { clínico }\end{array}$ & $\begin{array}{c}\text { Con } \\
\text { indicador } \\
\text { clínico }\end{array}$ \\
\hline \multirow{2}{*}{$\begin{array}{c}\text { Funcionalidad } \\
\text { familiar }\end{array}$} & $\begin{array}{c}\text { Funcional } \\
\text { Disfunción } \\
\text { moderada } \\
\text { Disfunción } \\
\text { severa }\end{array}$ & $31.7 \%$ & $7.9 \%$ \\
\hline \hline
\end{tabular}

Como se ilustra en la tabla 3, con respecto a las asociaciones entre el rendimiento académico y el reporte de los padres de la conducta internalizada de sus hijos adolescentes, el $6.3 \%$ de los adolescentes que evidencia comportamientos internalizados presenta un rendimiento académico medio mientras que un $7.9 \%$ presenta un rendimiento académico bajo, con una significancia de .02 .

Tabla 3. Asociación entre el rendimiento académico y las conductas internalizadas (CBCL)

\begin{tabular}{cccc}
\hline \hline & & \multicolumn{2}{c}{ Conductas internalizadas } \\
\cline { 3 - 4 } & & $\begin{array}{c}\text { Sin indicador } \\
\text { clínico }\end{array}$ & $\begin{array}{c}\text { Con indicador } \\
\text { clínico }\end{array}$ \\
\hline \hline \multirow{2}{*}{$\begin{array}{c}\text { Rendimiento } \\
\text { académico }\end{array}$} & Medio & $38.2 \%$ & $17.5 \%$ \\
& Bajo & $7.9 \%$ & $6.3 \%$ \\
\hline \hline
\end{tabular}


Por otro lado, en la tabla 4 se encuentra que según lo reportado por los padres, un $25.4 \%$ de los adolescentes con conductas internalizadas presenta una disfunción familiar moderada y un $3.2 \%$ evidencia una disfunción familiar moderada.

Tabla 4. Asociación entre la funcionalidad familiar y las conductas internalizadas (CBCL)

\begin{tabular}{cccc}
\hline \hline & & \multicolumn{2}{c}{$\begin{array}{c}\text { Conductas } \\
\text { internalizadas }\end{array}$} \\
\cline { 3 - 4 } & & $\begin{array}{c}\text { Sin } \\
\text { indicador } \\
\text { clínico }\end{array}$ & $\begin{array}{c}\text { Con } \\
\text { indicador } \\
\text { clínico }\end{array}$ \\
\hline Funcionalidad \\
familiar & $\begin{array}{c}\text { Funcional } \\
\text { Disfunción } \\
\text { moderada }\end{array}$ & $7.9 \%$ & $3.2 \%$ \\
& $\begin{array}{c}\text { Disfunción } \\
\text { severa }\end{array}$ & $12.7 \%$ & $3.2 \%$ \\
\hline \hline
\end{tabular}

De acuerdo con la tabla 5, respecto a las asociaciones entre el rendimiento académico y el reporte de los profesores sobre la conducta internalizada de los adolescentes, el $9.3 \%$ de los adolescentes con comportamientos internalizados presenta un rendimiento académico medio, mientras que un $3.2 \%$ presenta un rendimiento académico bajo, con una significancia de .503 .

Tabla 5. Asociación entre el rendimiento académico y las conductas internalizadas (TRF)

\begin{tabular}{cccc}
\hline \hline & & \multicolumn{2}{c}{ Conductas internalizadas } \\
\cline { 3 - 4 } & & $\begin{array}{c}\text { Sin } \\
\text { indicador } \\
\text { clínico }\end{array}$ & $\begin{array}{c}\text { Con } \\
\text { indicador } \\
\text { clínico }\end{array}$ \\
\hline \hline \multirow{2}{*}{$\begin{array}{c}\text { Rendimiento } \\
\text { académico }\end{array}$} & Alto & $8.7 \%$ & $15.2 \%$ \\
& Medio & $65.5 \%$ & $9.3 \%$ \\
\hline \hline
\end{tabular}

Por otro lado, en la tabla 6 se encuentra que según lo reportado por los profesores, un $3.2 \%$ de los adolescentes con conducta externalizada presenta una disfunción familiar moderada y otro 3.2 \% una disfunción familiar severa.
Tabla 6. Asociación entre la funcionalidad familiar y las conductas internalizadas (TRF)

\begin{tabular}{cccc}
\hline \hline & & \multicolumn{2}{c}{$\begin{array}{c}\text { Conductas } \\
\text { internalizadas }\end{array}$} \\
\cline { 3 - 4 } & & $\begin{array}{c}\text { Sin } \\
\text { indicador } \\
\text { clínico }\end{array}$ & $\begin{array}{c}\text { Con } \\
\text { indicador } \\
\text { clínico }\end{array}$ \\
\hline \hline \multirow{2}{*}{$\begin{array}{c}\text { Funcionalidad } \\
\text { familiar }\end{array}$} & $\begin{array}{c}\text { Funcional } \\
\text { Disfunción } \\
\text { moderada } \\
\text { Disfunción } \\
\text { severa }\end{array}$ & $31.7 \%$ & $6.8 \%$ \\
\hline \hline
\end{tabular}

\section{Discusión}

La presente investigación tuvo como finalidad identificar la asociación entre el nivel de funcionalidad familiar, las conductas internalizadas y el rendimiento académico en un grupo de adolescentes de un colegio público de la localidad de Usaquén en la ciudad de Bogotá, Colombia.

Los resultados van en la línea de lo obtenido en investigaciones previas que muestran diferencias en los factores internalizantes. De acuerdo a otros resultados obtenidos en el contexto colombiano (Vinaccia et al., 2006).

En lo encontrado en los resultados se evidenció disfuncionalidad familiar y un rendimiento académico medio, lo cual se puede relacionar con lo encontrado en otros estudios que indican la relación de los padres y la escuela como factores moderadores en la conducta internalizante (Cova, \& Maganto, 2005; Crawford, Schrock, \& Woodruff-Borden, 2011; Strijker, Van y Knot-Dickscheit, 2011).

A partir de lo observado en otras investigaciones la prevalencia de la ansiedad como un factor que se presenta con más frecuencia en las conductas internalizantes, de hecho esta conducta sobresale en los estudiantes afectando su rendimiento académico y funcionalidad familiar (Amézquita, González, \& Zuluaga, 2000). Con respecto a la depresión se evidencia que los adolescentes son susceptibles a la depresión con respecto a su situación familiar y escolar, esto les trae inconformidad la cual se refleja en sus conductas internalizantes tal cual como se observa en este estudio realizado (Aguilar, De Eduardo, \& Berganza, 1996). 
El análisis de los resultados del reporte para padres, mostró que aproximadamente un cuarto de la población evaluada presentó algún tipo de conducta internalizante, lo cual concuerda con el estudio de Hewitt et al. (2010) quienes encontraron una prevalencia en las conductas internalizadas de $25 \%$ en una localidad de la ciudad de Bogotá; problemática que, también según el Estudio Nacional de Salud Mental del Adolescente, muestra la mayor prevalencia en el contexto colombiano para los problemas internalizados como la ansiedad con un 5.5\% y la depresión mayor con 5 \% (Ministerio de Protección Social, Universidad CES y la Oficina de Naciones Unidas contra la Droga y el Delito - UNODC, 2010).

En cuanto al análisis de la asociación entre la funcionalidad familiar y las conductas internalizantes, los resultados arrojan que en un 28.6 \% de la población están presentes ambas variables; esto concuerda con el estudio de Barrera et al. (2006) quienes exponen que el porcentaje de los factores del medio familiar que tienen relación con la alteración conductual corresponde a un 50 \% siendo determinantes el tipo de estructura familiar, el bajo nivel de escolaridad de los padres, tutores y cuidadores.

Adicionalmente es de resaltar que el puntaje obtenido por medio del APGAR evidencia que más del $60 \%$ de la población presenta algún tipo de disfuncionalidad familiar, lo que de acuerdo con Gladding (1995) generaría otros problemas en la legitimidad de la autoridad, la estabilidad vs. flexibilidad, la participación, la adaptabilidad de sus integrantes lo que desembocaría en crisis.

Por otra parte, la asociación significativa entre rendimiento académico y conducta internalizada concuerda con López et al. (2010) y Hewitt et al. (2010) quienes postulan que el medio escolar es una fuente de imitación, socialización y transmisión del conocimiento tanto académico, como cultural, de normas y expectativas sociales por lo que ejecuta un rol relevante en las alteraciones de la conducta.

No obstante, en su análisis de regresión lineal, Aguilar et al. (1996) demostraron que cuando aumenta el valor de la autoestima académica, la depresión en los adolescentes disminuye; lo cual no se evidencia en el presente estudio, ya que la conducta internalizada, puntuó alto en rendimiento académico y en la presencia del problema. La divergencia entre los estudios pude deberse a que la relación con los compañeros y docentes, las responsabilidades académicas se asocian con la depresión, la ansiedad y las conductas suicidas (Amézquita et al., 2000).

Sin embargo, diferentes estudios coinciden en que la aplicación del (TRF) a profesores no es muy aplicada solo hace referencia al reporte de lista de chequeo a padres CBCL y el autorreporte YSQ para adolescentes, dando cumplimiento al objetivo en esta investigación que es describir la asociación entre la funcionalidad familiar, las conductas internalizadas y el rendimiento académico se tomó en cuenta el informe del profesor aplicando el TRF. Por otra parte, se sugiere para posteriores investigaciones sobre conductas externalizantes e internalizantes se presenten en todos los estratos socioeconómicos, y colegios tanto privados como públicos con un mayor número de participantes que implique la colaboración integrada de padres y profesores para prevenir estos factores internalizantes en niños y adolescentes.

\section{Conclusiones}

Los padres perciben mayores problemas internalizados en sus hijos que lo reportado por los adolescentes y los profesores. Esto podría estar relacionado con el hecho de que los adolescentes con conductas internalizadas tienden a presentar problemas en la funcionalidad familiar. Lo anterior concuerda con lo encontrado por Beyers, Bates, Pettit y Dodge (2003), quienes reportan que las conductas internalizadas no se presentan descontextualizadas, es decir, las influencias del ambiente ejercen un papel significativo en su manifestación, especialmente cuando es el ambiente familiar disarmónico el que se evidencia en la interacción entre padres e hijos.

En cuanto a las asociaciones halladas entre el rendimiento académico y la conducta internalizada, se puede decir que el entorno familiar ejerce un papel importante en el rendimiento académico de los hijos. Se revela una significativa relación entre el compromiso del padre en la educación de sus hijos y el rendimiento escolar, más allá del solo compromiso de la madre. La familia se siente angustiada cuando sus hijos no obtienen notas satisfactorias o esperadas, de igual manera, se podría decir que el estilo parental afecta el desempeño académico de los adolescentes, puesto que un estilo parental permisivo genera un nivel académico menor. De igual modo las interacciones que los adolescentes establecen 
con sus maestros pueden jugar un papel importante en el desarrollo o no de comportamientos internalizados.

De acuerdo a lo hallado en el estudio, se puede concluir que es necesario realizar un programa multicomponente integrado con los adolescentes, la familia y el colegio dirigido hacia el manejo de los problemas internalizados y hacia el fortalecimiento de la funcionalidad familiar, mediante estrategias que involucren entrenamiento en comunicación, solución de problemas, fortalecimiento de la autoestima y regulación emocional que contribuyan hacia el mejoramiento de las relaciones familiares y por otro lado, hacia la disminución de problemas de conducta interiorizados que redunden a su vez en el rendimiento académico de los adolescentes.

Este estudio se constituye en un insumo para el trabajo con la muestra evaluada, cuenta con la ventaja de tener la perspectiva de los propios adolescentes, los padres y los profesores sobre su comportamiento internalizado; a su vez permite tener un diagnóstico sobre la asociación de dicho comportamiento con la funcionalidad de la familia y el rendimiento académico de la muestra.

Moreno y Utria (2011) encontraron que los programas de tratamiento cognitivo conductual dirigidos a población infantil tienden a presentar falencias tanto en su planeación como en su implementación, en ese sentido se halló que en varios de estos programas no se realizó un diagnóstico previo, lo cual es fundamental para responder a las necesidades del grupo al cual se dirigen.

\section{Referencias}

Achenbach, T. (1985). Assesment and taxonomy of child adolescent psychopathology. London, England: Sage

Achenbach, T. M., \& Edelbrock, C. S. (2001). Manual for the Youth Self-Report and profile. Burlington, VT, University of Vermont Department of Psychiatry.

Achenbach, T., \& Rescorla, L. (2001). (A) Manual for the ASEBA School: Age Forms, \& Profiles. Child Behavior Checklist for Ages 6-18, Teacher's report from, youth self-report and integrated system of multi-informant assessment. Burlington: University of Vermont.

Achenbach, T., \& Rescorla, L. (2001). (B) Manual for the ASEBA School- Age Forms, \& Profiles. Burlington, VT: University of Vermont, Research Center for Children, Youth, \& families.
Achenbach, T. M. (2009). The Achenbach System of Empirically Based Assessment (ASEBA): Development, Findings, Theory, and Applications. Burlington, VT: University of Vermont, Research Center for Children, Youth, \& Families.

Aguilar, G., De Eduardo, H., \& Berganza, C. (1996). Autoestima y depresión en adolescentes guatemaltecos. Revista Latinoamericana de Psicología, 28(2), 341-366.

Amézquita, A., González, R., \& Zuluaga, D. (2000). Prevalencia de la depresión, ansiedad y comportamiento suicida en la población estudiantil de pregrado de la Universidad de Caldas. Revista Colombiana de Psiquiatría, 23(4), 341-353.

Barrera, D., Restrepo, C., Labrador, C., Niño, G., Díaz, D., Restrepo, D., Lamus, F., López de Mesa, C., \& Mancera, B. (2006). Medio familiar y entorno escolar: detonantes y antídotos de las conductas agresivas en niños en edad escolar. Persona y Bioética, 10(2), 99-107.

Beyers, J. M., Bates, J. E., Pettit, G. S., \& Dodge, K. A. (2003). Neighborhood structure, parenting processes and the development of youths' externalizing behaviors: A multilevel analysis. American Journal of Community Psychology, 31, 35-53.

Betancourt, D., \& Andrade, P. (2011). Control parental y problemas emocionales y de conducta en adolescentes. Revista Colombiana de Psicología, 20, 1, 27-41.

Buckholdt, K. E., Parra, G. R., \& Jobe-Shields, L. (2014). Intergenerational transmission of emotion dysregulation through parental invalidation of emotions: implications for adolescent internalizing and externalizing behaviors. Journal of Child and Family Studies, 23, 324-332. doi: 10.1007/s10826013-9768-4

Cova, S., \& Maganto, C. (2005). Diferencias de género en preadolescentes en la vulnerabilidad a presentar comportamientos desadaptativos en condiciones de adversidad familiar. Revista Clínica y Salud, 16(1), 91-106.

Crawford, N. A., Schrock, M., \& Woodruff-Borden, J. (2011). Child Internalizing Symptoms: Contributions of Child Temperament, Maternal Negative Affect, and Family Functioning. Child Psychiatry, \& Human Development, 42, 53-64. 
Forero, L., Avendaño, M., Duran, M., Duarte, Z., \& Campo, A. (2006). Consistencia interna y análisis de factores de la escala APAGAR para evaluar el funcionamiento familiar en estudiantes de básica secundaria. Revista Colombiana de Psiquiatría. 1, 25, 23-29.

Gladding, S. (1995). Family Therapy. History, Theory, and Practice. New Jersey: Prentice-Hall.

Henao, A. M., \& Mahecha, J. C. (2005). Ajuste psicológico según edad y género en niños y jóvenes de estrato socioeconómico 1 y 2 de Bogotá. Suma Psicológica, 12(2), 197-212.

Hewitt, N., Jaimes, S., Vera., L. A., \& Villa, M. C. (2012). Características psicométricas del cuestionario de comportamientos infantiles CBCL en niños y adolescentes colombianos. Trabajo de Grado. Facultad de Psicología. Universidad de San Buenaventura. Bogotá - Colombia.

Hewitt, N., Moreno, J., Oliveros, E., \& Pardo, A. (2010). Caracterización de comportamientos externalizados e internalizados en niños de 8 a 12 años de la localidad de Usaquén de la ciudad de Bogotá. Ponencia presentada en el $\mathrm{V}$ Congreso Nacional de Innovaciones en Psicología y Salud Mental. Tunja 13-17 de septiembre de 2010.

Jiménez, M. (2000). Competencia social: intervención preventiva en la escuela. Infancia y Sociedad, 24, 21 48.

Jiménez, T., Musitu, G., \& Murgui, S. (2005). Familia, apoyo social y conducta delictiva en la adolescencia: efectos directos y mediadores. Anuario de Psicología, 36(2), 559-579.

Lemos, V. (2003). La deseabilidad social en la evaluación de la personalidad infantil. Trabajo presentado en el IV Congreso Iberoamericano de Evaluación Psicológica. AIDEP. Lima, Perú.

López, C., Alcántara, M., Fernández, V., Castro, M., \& López Pina, J. (2010). Características y prevalencia de los problemas de ansiedad, depresión y quejas somáticas en una muestra clínica infantil de 8 a 12 años, mediante el CBCL (Child Behavior Checklist). Anales de Psicología, 26(2), 325 - 334.

McCullough, C., \& Shaffer. A. (2014). Maternal depressive symptoms and child externalizing problems: moderating effects of emotionally maltreating parenting behaviors Journal of Child and
Family Studies, 23, 389-398. doi 10.1007/s10826013-9804-4

Ministerio de Protección Social, Universidad CES y la Oficina de Naciones Unidas contra la Droga y el Delito - UNODC (2010). Situación de la salud mental del adolescente, Estudio Nacional de la Salud Mental Colombia. Bogotá.

Moreno, J., \& Chauta, L. (2012). Funcionalidad familiar, conductas externalizadas y rendimiento académico en un grupo de adolescentes de la ciudad de Bogotá. Psychologia. Avances de la Disciplina, 6, 1, 155-166.

Moreno, J., \& Utria, O. (2011). Caracterización de los programas de tratamiento cognitivo-conductual para el manejo de problemas de comportamiento en niños y adolescentes realizados en Bogotá entre 2002 y 2008. Psicología desde El Caribe, 28, 39-76.

Palacios, J., \& Rodrigo, M. J. (1998). La familia como contexto de desarrollo humano. En M. J. Rodrigo y J. Palacios (Eds.). Familia y desarrollo humano. (pp. 25-38). Madrid: Alianza.

Paz, S. (2007). Problemas en el desempeño escolar y su relación con su funcionalismo familiar en alumnos de EGB1. Revista de la Facultad de Medicina, 8(1), 27-32.

Pérez, M. A., Pinzón-Pérez, H., \& Alonso, L. M. (2007). Aplicaciones de la promoción de la salud: Dinámica familiar y riesgos. En M. A. Pérez, H. Pinzón-Pérez y L. M. Alonso (Ed.). Promoción de la salud. Conceptos y aplicaciones. (pp. 93-115) Barranquilla: Uninorte.

Portellano, A. (1989). Fracaso escolar. Diagnóstico e intervención. Una perspectiva neuropsicológica. Madrid: Ciencias de la educación preescolar y especial.

Rescorla, L., Ivanova, M., Achenbach, T., Begovac, I., Chahed, M. et al. (2012). International Epidemiology of Child and Adolescent Psychopathology: Integration and Applications of Dimensional Findings from 44 Societies. Journal of the American Academy of Child and Adolescent Psychiatry, 51, (12), 1273-1283. doi: 10.1016/j. jaac.2012.09.012.

Sattler, J. (2003). Evaluación infantil: aplicaciones cognitivas. México: El Manual Moderno. 
Smilkstein, G. (1978). The family APGAR: a proposal for a family function test and its use by physicians. Journal of Family Practice, 6(6), 1231-1239.

Strijker, J., Van, S., \& Knot-Dickscheit, J. (2011). Assessment of problem behavior by foster parents and their foster children. Child and Family Social Work, 16(1), 93-100.

Vinaccia, S., Gaviria, A., Atehortúa, L., Martínez, P., Trujillo, C., \& Quiceno, J. (2006). Prevalencia de depresión en niños escolarizados entre 8 y 12 años del oriente antioqueño a partir del child depression inventory (CDI). Revista Perspectiva en Psicología, 2(1), 217 - 227.

Viola, L. (2010). Repercusión de la violencia doméstica en la salud mental infantil. Valoración del daño físico. Revista de Psiquiatría del Uruguay, 74(1), 7383. 